\title{
"Mycotic" coronary artery aneurysm and purulent pericarditis: The riddle of the chicken and the egg
}

Panteleimon Papakonstantinou ${ }^{1}$, Pantelis Gounopoulos ${ }^{1}$, Achilles Zacharoulis ${ }^{1}$, Eleni Papagianni $^{1}$, Konstantinos Papakonstantinou ${ }^{1}$, Terspichori Fragkouli ${ }^{1}$, and Antonios Sideris ${ }^{1}$

${ }^{1}$ Evangelismos Athens General Hospital

March 19, 2021

\begin{abstract}
Infected coronary artery aneurysms present high mortality and surgical management is the treatment of choice in most cases. We present a case of a giant infected aneurysm of the mid right coronary artery complicated with purulent pericarditis in an 83-year-old male patient. It is unknown whether the aneurysm or purulent pericarditis preceded. The patient underwent urgent aneurysm resection and coronary artery distal bypass grafting. He died 24 hours after the operation. When purulent pericarditis and ICAA co-exist the riddle of the chicken and the egg becomes apparent.
\end{abstract}




\section{Introduction}

Purulent bacterial pericarditis is uncommon, accounting for $<1 \%$ of cases $^{1}$. It is generally manifested as a serious febrile disease, which should be managed aggressively with urgent pericardiocentesis, as death is inevitable if it remains untreated ${ }^{1}$. Giant "mycotic" coronary artery aneurysms (over $2 \mathrm{~cm}$ in diameter) ${ }^{2}$ are rare but potentially fatal infectious conditions usually associated with infectious endocarditis or bacteremia, particularly in immune-compromised patients ${ }^{3,4}$. The term "mycotic" is a misnomer but is commonly used to describe an infected coronary artery aneurysm (ICAA) regardless of the type of infection ${ }^{4}$. Herein, we present a case of a giant infected aneurysm of the right coronary artery (RCA) complicated with purulent pericarditis in an 83-year-old male patient.

\section{Case Description}

An 83-year-old male patient was referred to the cardiology department of our hospital due to acute myopericarditis. His medical history included hypertension, diabetes mellitus, dyslipidemia, permanent atrial fibrillation, hyperuricemia, and benign prostatic hyperplasia.

On admission, the patient was afebrile and hemodynamically stable. Laboratory tests showed high white blood cells (WBC) of $21.090 \mathrm{~K} / \mu \mathrm{L}$, elevated level of C-reactive protein (CRP) of $7.4 \mathrm{mg} / \mathrm{dL}$ [upper limit of normal (ULN): $0.5 \mathrm{mg} / \mathrm{dL}$ ], erythrocyte sedimentation rate of $120 \mathrm{~mm} / \mathrm{h}$ and an impaired renal function of $44 \mathrm{ml} / \mathrm{min} / 1.73 \mathrm{~m}^{2}$ [Glomerular Filtration Rate by the MDRD Equation- Creatinine level: $1.6 \mathrm{mg} / \mathrm{dL}$ ]. High sensitive Troponin-T was elevated [132 pg/ml (ULN: $12 \mathrm{pg} / \mathrm{ml}$ )]. The QuantiFERON-TB Gold In Tube test was negative for Mycobacterium tuberculosis infection. Virological blood test for herpesviruses IgM (cytomegalovirus, Epstein-Barr virus), enteroviruses IgM (echoviruses, coxsackieviruses), adenoviruses IgM, 
parvovirus IgM and human immunodeficiency virus were negative. COVID-19 (SARS-CoV-2) test was also negative. Thyroid hormones were within the normal limits. Rheumatoid factor, anti-nuclear antibodies and anti-neutrophil cytoplasmic antibodies were negative.

Echocardiography revealed a moderate circumferential pericardial effusion. Left ventricle had a normal systolic function, while the right ventricle had impaired systolic function [Decreased triscuid annular plane systolic excursion (TAPSE) measured with M-mode $(7 \mathrm{~mm})$ and decreased peak systolic (S') velocity of triscuid annulus $(7 \mathrm{~cm} / \mathrm{s}$ ). Flattened interventricular septum (D-shaped left ventricle) was also observed at the parasternal short axis view. Inferior vena cava was distended $(27 \mathrm{~mm})$ with diminished inspiratory collapsibility $(<30 \%)$ (subcostal view). Subcostal view revealed a cyst-like lesion about 3.7 X $3.5 \mathrm{~cm}$ over the right atrioventricular groove with thickened walls and within it a calcified spherical formation (Fig. 1B ). After the intravenous injection of an ultrasound contrast agent there was a delayed entry of the contrast agent into the lesion (Supplemental Video 1 ). Chest computed tomography showed pericardial effusion and a lesion at the mid right coronary artery (RCA) (Fig. 1A ). Coronary angiography revealed a giant aneurysm of the mid-RCA (Fig. 1C) with extravasation of the contrast media (Supplemental Video 2 ). The patient underwent an urgent surgical aneurysm resection and coronary artery distal bypass grafting. After the sternotomy, the pericardium was noted to be extremely inflamed with the presence of frank pus in the pericardial cavity (Fig. 1D) . Intraoperatively, RCA aneurysm was ruptured, while a bypass grafting of the RCA was performed with a saphenous vein graft. The patient died about 24 hours after the operation due to hemodynamic instability. Pericardial fluid cultures grew methicillin-sensitive Staphylococcus aureus .

The Histology (Fig. 2 ) revealed abundant thrombotic material, containing red blood cells and condensing fibrin, with a necrotic and partially hyalinised vascular wall (coronary artery) locally heavily infiltrated by transmural inflammatory cells. More recent thrombus elements were observed on the luminal surface of the artery (Fig. 2A, C ). Immunostaining for smooth muscle actin (SMA) showed markedly decreased or totally absent staining for SMA, due to loss of smooth muscle cells (Fig. 2B ). Immunostaing for elastin fibers (Elastica IHC) showed complete loss of elastic fibers. Numerous bacterial aggregates (clumps) were detected in Hematoxylin-Eosin (H-E) stain (Fig. 2C) and identified by Gram stain (Fig. 2D ). The histopathology was consistent with a "mycotic" (infected) aneurysm of the mid-RCA.

\section{Discussion}

Bacterial infection of the pericardial space is an uncommon cause of pericardial effusion ${ }^{1}$. Infected coronary artery aneurysms are rare and they occur mostly after stent placement or in septic and immune-compromised patients. However, spontaneous ICAAs have been reported previously ${ }^{3}$, as in our case. The literature of ICAA is mainly based on case reports or case series ${ }^{4,5}$. The clinical course of coronary artery aneurysm remains largely unpredictable and without appropriate diagnosis and treatment the outcome is invariably poor with sepsis and aneurysmal rupture with resultant hemodynamic collapse and death ${ }^{4}$. Exceedingly high morbidity and mortality have been reported (mortality $43-53 \%)^{5}$. Surgical management was the treatment of choice for the most ICAAs. The most common surgical method is ICAA resection and distal bypass of the artery (mortality rate of $20.9 \%)^{5}$.

Our case is also uncommon in that the presentation involved purulent pericarditis. It is unknown whether the aneurysm or purulent pericarditis preceded. The impaired systolic function of the right ventricle can be attributed to the myocardial ischemia of the right ventricle due to the RCA aneurysm. Consequently, we can not determine if the ICAA was the cause of purulent pericarditis (ICAA leak into the pericardial sac) or the result of the purulent pericarditis (local injury of the CAA due to the artery's wall inflammation and artery's wall bacterial infection from pericardial fluid). Therefore, safe conclusions about the onset of purulent pericarditis and the formation of the CAA can not be drawn.

In conclusion, this was a rare case of a spontaneous giant "mycotic" aneurysm of the RCA presented with purulent pericarditis. ICAAs present high mortality and surgical management is the treatment of choice in most cases. When purulent pericarditis and ICAA co-exist the riddle of the chicken and the egg becomes apparent. 


\section{References}

1. Adler Y, Charron P, Imazio M, et al. 2015 ESC Guidelines for the diagnosis and management of pericardial diseases: The Task Force for the Diagnosis and Management of Pericardial Diseases of the European Society of Cardiology (ESC)Endorsed by: The European Association for Cardio-Thoracic Surgery (EACTS). European heart journal.2015;36(42):2921-2964.

2. Arcinas LA, Yan W, Jassal DS, Love MP, Yamashita MH, Elbarouni B. Multimodality Imaging of a Giant Right Coronary Artery Aneurysm. The Canadian journal of cardiology.2018;34(12):1688.e1685-1688.e1687.

3. Borgi JF, Natrajan KM, Sun JC. Mycotic aneurysm of the right coronary artery presenting as infected pericardial effusion. Circulation.2014;130(2):e7-8.

4. Restrepo CS, Gonzalez TV, Baxi A, Rojas CA. Infected ("Mycotic") coronary artery aneurysm: Systematic review. Journal of cardiovascular computed tomography. 2020;14(6):e99-e104.

5. Baker DW, Whitehead NJ, Barlow M. Mycotic Coronary Aneurysms.Heart, lung ES circulation. 2020;29(1):128-136.

\section{FIGURE LEGENDS}

\section{Figure 1}

A: Chest computed tomography: Round formation in the mid right coronary artery (arrows) with eccentric calcification. Pericardial effusion of increased density (star). Right pleural effusion (arrowhead).

B: Subcostal echocardiographic view. Right atrial enlargement (triangle); cyst-like lesion about 3.7 X 3.5 $\mathrm{cm}$ over the right atrioventricular groove (star) and within it a calcified spherical formation (arrow).

C: Coronary angiography: Aneurysm of the mid right coronary artery.

D: Intraoperative image. Frank pus in the pericardial cavity after the pericardiotomy.

\section{Figure 2}

A : Thrombotic material and a necrotic vascular wall (original magnification X40, Hematoxylin-Eosin (H-E) stain, objective 4x, OLYMPUS microscope BX41)

B : Smooth muscle actin (SMA) positivity in smooth muscle cells (original magnification X40, SMA immunostain / DAKO SMA monoclonal antibody, objective 4x, OLYMPUS microscope BX41)

C : Vascular wall with bacterial formations (original magnification X100, H-E stain, objective 10x, OLYMPUS microscope BX41)

D : Bacterial formations. Gram-positive stain (original magnification X400, Gram stain, objective 40x, OLYMPUS microscope BX41)

\section{VIDEO LEGENS}

Video 1. Echocardiographic subcostal view. Mid- right coronary aneurysm after the infusion of intravenous contrast agent.

Video 2. Mid right coronary artery aneurysm leak into the pericardial sac.

\section{FIGURES}

Figure 1. 
Figure 2 . 
